DETC2002/MECH-34317

\title{
A FINGER MECHANISM FOR ADAPTIVE END EFFECTORS
}

\author{
Venketesh N Dubey \\ School of Design Engineering and Computing \\ Bournemouth University \\ 12 Christchurch Road, Studland House \\ Bournemouth, BH1 3NA, UK \\ Tel. +441202503791 \\ Fax. +441202503751 \\ Email: vdubey@bmth.ac.uk
}

\author{
Richard M Crowder \\ Intelligence Agents Multimedia Group \\ Department of Electronics and Computer Science \\ University of Southampton \\ Southampton, SO17 1BJ, UK \\ Tel. +4423 80593441 \\ Fax. +442380592865 \\ Email: rmc@ecs.soton.ac.uk
}

\begin{abstract}
This paper presents design and analysis of a rigid link finger, which may be suitable for a number of adaptive end effectors. The design has evolved from an industrial need for a tele-operated system to be used in nuclear environments. The end effector is designed to assist repair work in nuclear reactors during retrieval operation, particularly for the purpose of grasping objects of various shape, size and mass. The work is based on the University of Southampton's Whole Arm Manipulator, which has a special design consideration for safety and flexibility. The paper discusses kinematic issues associated with the finger design, and to the end of the paper specifies the limits of finger operating parameters for implementing control laws.
\end{abstract}

Keywords: End Effectors, Finger Design, Robotic Hand, Adaptive Finger, Mechanism Analysis

\section{INTRODUCTION}

Dexterous manipulation is an area of robotics where an end effector with co-operating multiple fingers is capable of grasping and manipulating an object. One of the main characteristics of the dexterous manipulation concept is that it is object centered. As dexterous manipulation is quintessentially a human activity, majority of the dexterous robotic end effectors developed to date has considerable anthropomorphic characteristics. In view of the importance of the research area, a considerable body of research literature is available on the analysis of grasp quality and control of the end effectors. Review by Okamura et al provides an excellent introduction to the field [1]. However, very few papers address the issues associated with end effector design and its evolution for specific application needs, particularly computation of loading condition of the finger components for a known fingertip interaction to ensure robust mechanical design. Further to achieve a specific grip, study of the finger motion is important to validate design as well as for implementing position control algorithms.

The finger mechanism presented in this paper originated with the design developed for the University of Southampton Whole Arm Manipulator (WAM) [2]. This manipulator was developed for insertion into the human sized hyperlon ${ }^{1}$ glove for use in a conventional glove box. Due to the design requirement, this manipulator has an anthropomorphic end effector with four adaptive fingers and a prehensile thumb, the grasp being controlled by three motor-gearboxes assembles located within the palm, with connections to the finger segments via solids mechanical linkages. The WAM design

${ }^{1}$ Hyperlon is the trade name of a high performance rubber used in specialist applications. 
rational was dictated by the tight size constraint of the hand, its enclosing glove and the operating environment. The WAM's hand is capable of forming a range of grasps, which allowed it to perform a wide range of handling operations. However due to the design of the finger mechanism, the movement of its fingers cannot be precisely controlled during grasping operation, as this is determined by the compliance of the glove. Subsequent to the WAM design, a three-fingered end effector has been developed [3] based on a modified finger design; it is this design that is analyzed in this paper.

The gripper design process starts with a review of grasp taxonomy, relating the required task to the available grasps. It is clear that the hand kinematics and forces are closely related to the grip postures. As detailed by Cutkosky [4], two main grip classifications can be identified, either as a precision or power grip. In a precision grip, contact is made at the tip of the finger, while in the power grip the fingers enclose the object and fully constrain it. In a power grip the grasped object is constrained by multiple contacts between the object and the fingers [5] with no forces being transmitted via the top finger segment.

In a pre-grasp situation for known shape and size of the object, fingers are required to move to precise locations in a coordinated manner to form a secure grasp. This requires an accurate knowledge of the finger kinematics. Also for design purpose, torque transmission through the finger linkages and estimation of the loading condition of finger components are of important consideration for safety and reliability, if it has to operate in a nuclear environment. Thus kinematic and static analyses of the finger mechanism are considered in this paper for design of the multi-fingered end effectors with articulated links.

\section{FINGER DESIGN}

Over the last thirty years a considerable number of dexterous end effectors have been developed. The most notably being the Stanford/JPL [6] and Utah/MIT hands [7], that were developed to research object manipulation. These designs are based on the fingers being actuated via tendons from an external actuator pack. The Belgrade/USC hand [8] was developed with prosthetic application in mind, and has a more compact actuating mechanism. Okada [9] designed a threefingered hand with 11 degrees of freedom using pulley/tendon system to perform assembly operations. Another three-fingered hand developed at the University of Pennsylvania [10] and later marketed as BarrettHand has a compact design, however, the hand uses four actuators on a worm drive with cable and breakaway clutch to provide finger motions. Other notable hands include the Karlsruhe hand [11], NTU hand [12] and Delft University of Technology hand [13]. The design of the hand and finger are to a large extent dictated by the approach taken to transmit the actuator forces to the finger joint. If special purpose localized actuators, such as artificial muscles are excluded, only two realistic approaches for power transmission within the hand between the finger-joints and actuators need to be considered; tendons or a rigid link kinematic chain.

\section{Tendon vs. Solid Drives}

Many dexterous hand designs are tendon based, where each finger joint is connected to a remote actuator by a flexible cord or tendon. To achieve full joint motion a minimum of two tendons are required per joint. The advantage of this approach is that the actuators are remote from the hand and hence reducing the overall inertia by removing mass from the end of the manipulator. If size is not a limitation, the actuators can be mounted external to the hand, with the power transmission to the hand via tendons. While satisfactory for experimental systems, this approach is not suitable for industrial applications. The space restriction imposed by certain industrial applications result in the external actuators together with tendons not being a practical design proposition. In addition, the use of hand mounted pneumatic and hydraulic actuators in many applications are considered to be impractical, due to leakage problems. In a number of applications for a fully dexterous hand to operate satisfactorily, electric actuators need to be located within the profile of the end effector. As physical size of the system limits the number of actuators, the design solution presented requires the motion of the fingers to be controlled by solid mechanical linkages. An advantage of this design is the high reliability of electric motors; this was an important consideration as the manipulator is intended for continuous industrial operation.

\section{Finger Mechanism}

The finger mechanism consists of three sections (lower, middle and tip) pivoted together as shown in Fig. 1, with the maximum relative movements of $90^{\circ}$ between each section.

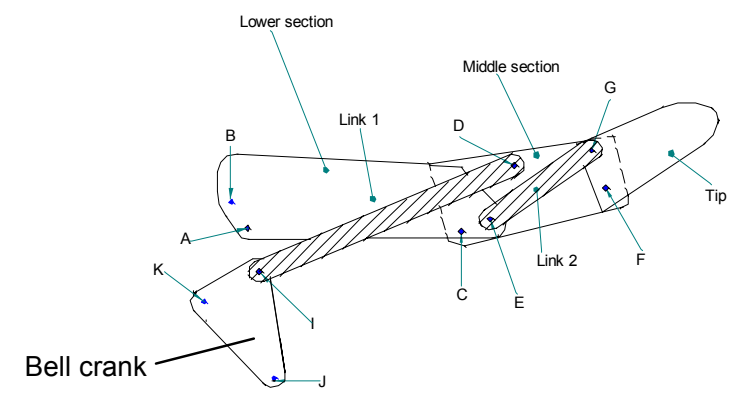

Fig. 1. Basic Finger Mechanism (bell crank displaced)

The upper two finger sections are used to produce a coordinated curling motion. The tip is linked to the lower section by link 2 , so that any motion of the middle section by link 1 will cause the tip to move, producing curling motion to the finger. The lower and middle sections are individually connected to the actuating mechanism at points $\mathrm{B}$ and $\mathrm{K}$. The mechanism is grounded at joints $\mathrm{A}$ and $\mathrm{J}$.

The finger mechanism can be considered to have two degrees of freedom:

- Bending, where displacement of joint B bends all the three finger segments about joint $\mathrm{A}$.

- Curling, where displacement of joint $\mathrm{K}$ results in the curling of the two upper finger segments about joint $\mathrm{C}$.

Thus the finger discussed above requires two drive inputs. This can be produced in a number of ways, depending on the application requirements. Three approaches can be considered to achieve this: 
- Equalizing mechanism.

- Single motor, and differential gear box

- Fully independent drives.

\section{Equalizing Mechanism}

The equalizing bar mechanism used in the end effector of the Whole Arm Manipulator is shown in Fig. 2. In the rest position the finger is considered to be in the fully extended position. To close the finger the equalizer bar is driven to the left, by a crank and slider mechanism. Due to the built-in difference in the mechanical advantages between links A and B and their respective sections, the finger is designed to preferentially rotate around pivot A of the lower section. The design of the mechanism is such that the finger will remain straight while it rotates around this pivot. The rotation of the complete finger will continue until such time as the lower section is stopped either at its mechanical limit or by an external object.

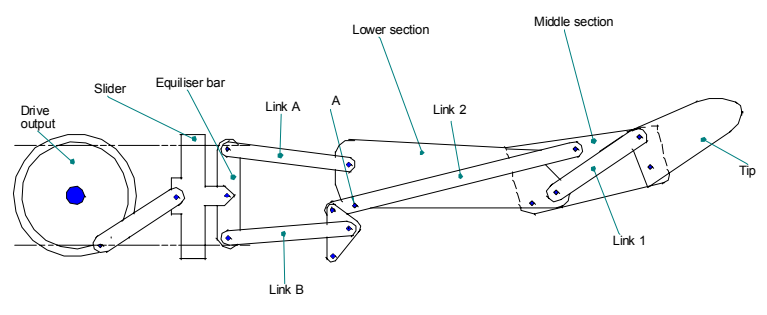

Fig. 2. Equalizing bar mechanism

As the lower section and link A cannot move, force is transferred to the middle section, via link $\mathrm{B}$, thus causing the upper two sections of the finger to curl over and complete the grip around the object. The resultant finger motion is similar to that of a human finger and is described as being "tip driven", as the fingertip effectively leads the motion. The position of the equalizing bar is controlled by the loads applied to the finger section thus is considered to be indeterminate. While compact, the design relies on external forces provided in the WAM's application by the hyperlon glove, which is used to stabilize the finger position.

\section{Differential Gearbox}

To satisfactorily control the finger for an adaptive end effector without the requirements of a compliant glove or similar systems, both input links need to be individually and positively controlled. This requirement led to the development of a mechanism capable of independently controlling the two input links. Due to application constraints imposed, only a single motor could be used to control all the required motions. The design of complete finger module can be considered in two parts; the finger, Fig. 3 and its actuating mechanism, Fig. 4.

The actuating mechanism has a central differential gearbox unit driving two lead screws supported on a rotary frame. The motor connected to the differential gearbox can be used to drive the two lead screws as well as the mechanism frame, providing three different components of a motion. These motions are determined by the use of three electromagnetic brakes.

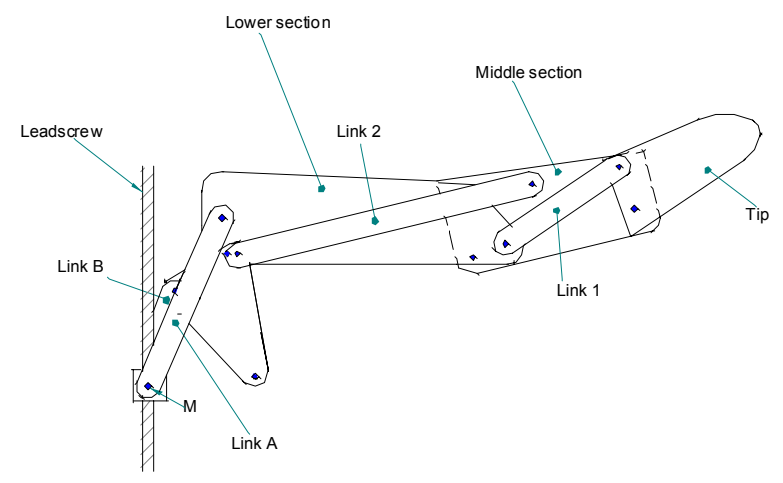

Fig. 3. Lead screw driven finger

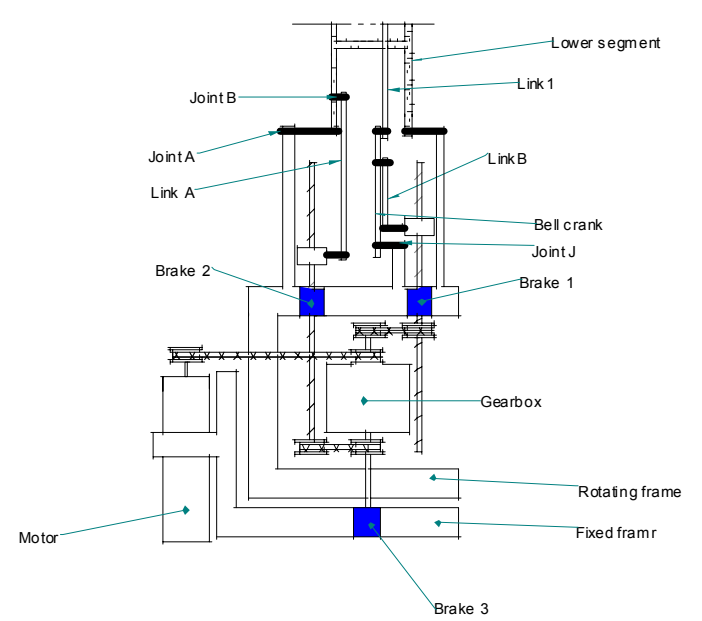

Fig. 4. Finger drive mechanism with differential gearbox

As shown in Fig. 4, brakes 1 and 2 control the two lead screws, while brake 3 controls the finger orientation relative to the end effector frame. Thus this mechanism provides three degrees of freedom to the finger. The structure of the finger offers independent curl motion while the bend motion is only partially independent as it results in a slight curling effect to the finger. By controlling the three brakes as shown in Table $1(\mathrm{R}$ signifies the brake release), the three components of motions can be controlled individually or in combination, thus operating the finger either in adaptive or precise control mode with concentric or opposing-thumb configuration.

Table 1. Finger motion controlled by the actuator brakes

\begin{tabular}{cccc}
\hline Brake 1 & Brake 2 & Brake 3 & Motion \\
\hline $\mathrm{R}$ & & & Curl \\
& $\mathrm{R}$ & & Bend \\
& & $\mathrm{R}$ & Rotate \\
\hline
\end{tabular}

As shown in Fig. 5, a prototype mechanism has been built and used to verify the performance of the transmission system. 


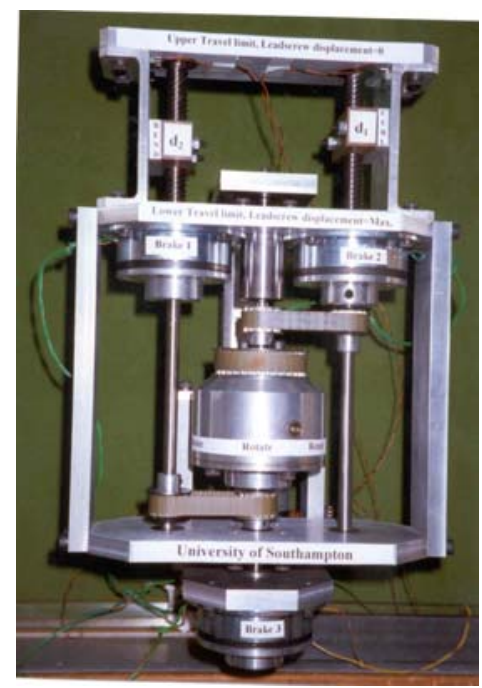

Fig. 5. Prototype finger drive mechanism

\section{Fully Controlled Mechanism}

This approach is the logical extension of the previous design, where all three motions are independently powered and controlled. This requires three motors within the end effector envelope for each finger. This approach does allow fully controlled independent motion to be achieved, however at the cost of additional cabling and possible size restrictions.

\section{Finger and End Effector Construction}

The basic construction of the finger is based on an open structure using side plates and cross pivots joining them. This mode of construction gives the maximum clear space within the profile of finger for accommodating the mechanical linkages and for incorporating any sensors within. In the end-effector design three individual fingers as shown in Fig. 6 are symmetrically placed, this gives a high degree of flexibility in gripping objects. As nine-degrees of freedom result form the design, a large number of precision and power grips can be produced. The flexibility of the hand is enhanced by the capability of the fingers to rotate about its own axis allowing generation of either two or three fingered parallel grips, or three fingered pinch grips [14].

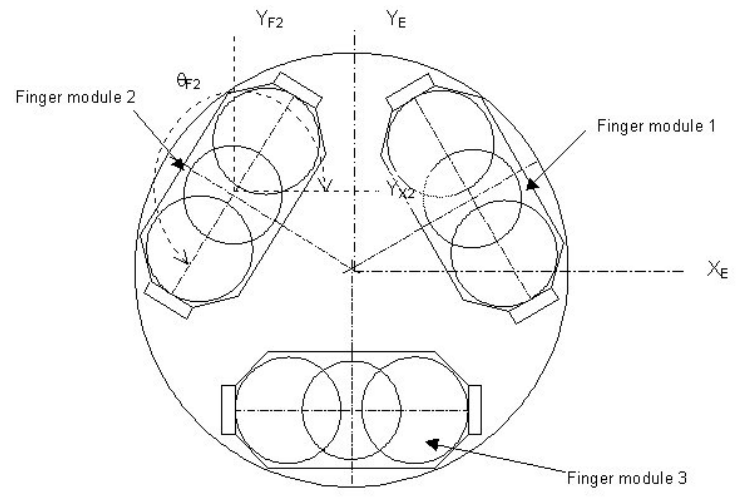

Fig. 6. Plan view of the three-fingered end effector

\section{FINGER KINEMATICS}

While homogenous matrix transformation has generally been used to represent the kinematic relation of the articulated links [15] and static force analysis for multi-fingered grasping [6], the present analysis is based on the vectorial method of link representation for developing static force and kinematic relationships.

The kinematic chain of the finger is shown in Fig. 7 with frames fixed to the finger joints. The fingertip position is given by,

$$
\begin{aligned}
& A H_{x}=\mathrm{a} \cos \alpha+\mathrm{b} \cos \beta+\mathrm{g} \cos \rho \\
& A H_{z}=\mathrm{a} \sin \alpha+\mathrm{b} \sin \beta+\mathrm{g} \sin \rho
\end{aligned}
$$

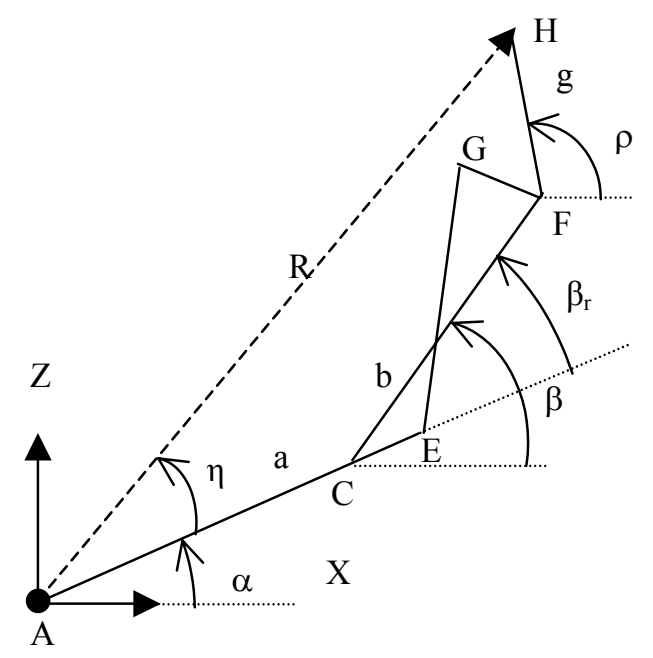

Fig. 7. Kinematic chain of the finger

The solution for these angles develops into complex nonlinear relations with many inverse trigonometric functions [14]. These relationships describe a closed form kinematic relation between two lead screw inputs (defined as $d 1, d 2$ ) and the fingertip position. From these relations fingertip workspace with reference to the finger's datum over the displacement range of lead screw can be plotted as is shown in Fig. 8 .

\section{Inverse Kinematics Solution}

Further to move the fingertip to a specified location, displacement of the lead screws needs to be known in advance. The inverse solution for such a mechanism should have fast convergence, and must operate satisfactorily throughout the finger workspace.

Existing end effectors have either adaptable fingers [2,8], which do not require exact inverse kinematics, or the finger movement is determined by the interpolation of the joint positions between two successive locations $[6,7,9]$. The movement of finger to a precise point in case of adaptable configurations has proved to be difficult whereas the exact tracking of a finger trajectory in rest cases may prove to be time consuming due to the interpolating nature of the implementation. The numerical inverse kinematic solution 
presented in this paper is considered to be fast enough to be tenable to real-time applications.

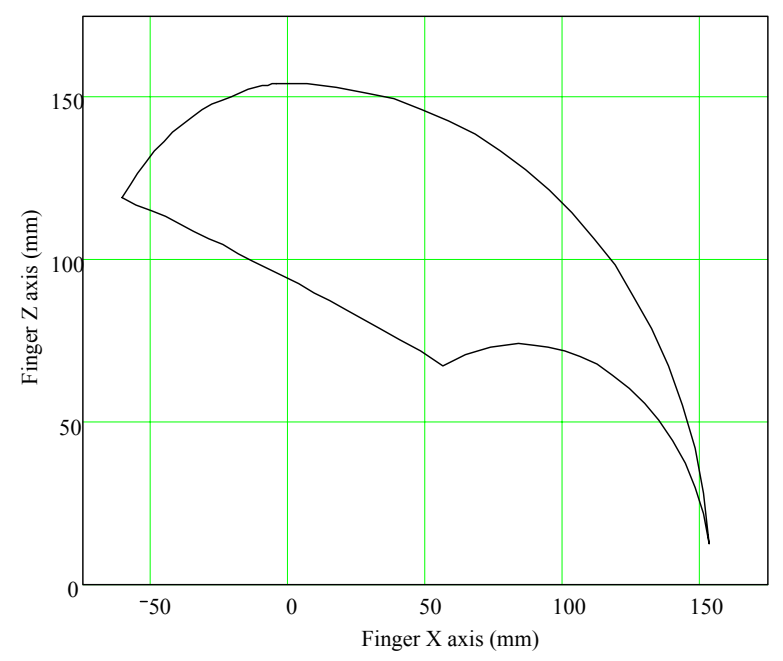

Fig. 8. Loci of the fingertip over lead screw travel limits

\section{Solution Strategy}

The numerical solution is based on the calculation of the two fingers angles, $\alpha$ and $\beta$, for a given fingertip location $H$, as shown in Fig. 7. These are selected since angles $\alpha$ and $\beta$ determine the bend and curl motion of the finger. In order to bring point $\mathrm{H}$ to a target point $\mathrm{P}$, the finger is first assumed to be driven in curl until the radius $\mathrm{AH}$ equaled the distance from A to the target point, then the finger is bent to bring point $\mathrm{H}$ to the target point. This technique helps in separating curl and bend angles, which allows an easy derivation of the displacement components. Once these angles are known, lead screw displacements $d 1$ and $d 2$ can be back calculated. An independent curl variable $\beta_{\mathrm{r}}$ ( $\beta$ relative to $\alpha$ ) is defined which equals $(\beta-\alpha)$, and the angle $\alpha$ is set to zero. The fingertip location can now be represented in polar form using $R$ and $\eta$ as shown in Fig. 7.

Under this situation all the finger variables can be solely represented as a function of the single variable $\beta_{\mathrm{r}}$ therefore,

$$
\begin{aligned}
& \mathrm{R}=\mathrm{f}_{1}\left(\beta_{\mathrm{r}}\right)=\sqrt{A H_{\mathrm{xr}}^{2}+A H_{\mathrm{zr}}^{2}} \\
& \eta=\mathrm{f}_{2}\left(\beta_{\mathrm{r}}\right)=\tan ^{-1}\left\{\frac{\mathrm{AH} \mathrm{zr}_{\mathrm{rr}}}{\mathrm{AH_{xr }}}\right\}
\end{aligned}
$$

Where $\mathrm{AH}_{\mathrm{xr}}$ and $\mathrm{AH}_{\mathrm{zr}}$ are the $x$ and $z$ components of the vector $\mathrm{AH}$. For a given target point $\mathrm{P}$ with respect to the joint $\mathrm{A}$, the polar form of position vector can be written as,

$$
\begin{aligned}
& |\mathrm{AP}|=\sqrt{\left(\mathrm{AP}_{\mathrm{x}}^{2}+\mathrm{AP}_{\mathrm{z}}^{2}\right)} \\
& \angle \mathrm{AP}=\tan ^{-1}\left\{\frac{\mathrm{AP}_{\mathrm{z}}}{\mathrm{AP}_{\mathrm{x}}}\right\}
\end{aligned}
$$

At the target point, the magnitude of the position vector in Eq. (3) and (5) are equal, hence

$$
\mathrm{AH}_{\mathrm{xr}}^{2}+\mathrm{AH}_{\mathrm{zr}}^{2}=\mathrm{AP}_{\mathrm{x}}^{2}+\mathrm{AP}_{\mathrm{z}}^{2}
$$

Since the target point is known, Eq. (7) can be written as,

$$
\mathrm{AH}_{\mathrm{xr}}^{2}+\mathrm{AH}_{\mathrm{zr}}^{2}-\mathrm{K}=0
$$

Where $\mathrm{K}$ is a constant, hence Eq. (8) can be solely expressed as function of $\beta_{\mathrm{r}}$

$$
\Rightarrow \mathrm{f}\left(\beta_{\mathrm{r}}\right)=0
$$

Eq. (9) can now be solved for $\beta_{\mathrm{r}}$. Once this is known, angle $\eta$ can be determined since this is a function of $\beta_{\mathrm{r}}$. The value of angle $\alpha$ can be defined as:

$$
\begin{aligned}
& \alpha=\angle \mathrm{AP}-\eta \\
& \Rightarrow \beta=\beta_{\mathrm{r}}+\alpha
\end{aligned}
$$

Eq. (9) can be optimized for fast numerical solutions using the Newton-Raphson method,

$$
\beta_{\mathrm{r} 2}=\beta_{\mathrm{r} 1}-\frac{\mathrm{f}\left(\beta_{\mathrm{r} 1}\right)}{\mathrm{f}^{\prime}\left(\beta_{\mathrm{r} 1}\right)}
$$

Where $\beta_{\mathrm{r} 1}$ is the first approximation, $\beta_{\mathrm{r} 2}$ is a better approximation and $\mathrm{f}^{\prime}\left(\beta_{\mathrm{r} 1}\right)$ is the derivative of the function $\beta_{\mathrm{r} 1}$. Although the first approximation can be taken arbitrarily between $\beta_{r_{\text {min }}}$ to $\beta_{\mathrm{r}_{\text {max }}}$ values, however, for fast convergence the first approximation based on the following polynomial fitted to $\mathrm{f}\left(\beta_{\mathrm{r}}\right)$ is used.

$$
\left|\mathrm{AH}_{\mathrm{r}}\right|^{2}=\mathrm{a}_{1} \beta_{\mathrm{r}}^{2}+\mathrm{b}_{1} \beta_{\mathrm{r}}+\mathrm{c}_{1}
$$

Where coefficient $a_{1}, b_{1}$ and $c_{1}$ are constant for a specified finger linkages dimensions and can be determined using three known finger locations for known values of $\beta_{\mathrm{r}}$ in the operating range. Once these coefficients are known, for any target point the first approximation can be found by solving the following equation,

$$
\mathrm{a}_{1} \beta_{\mathrm{r}}^{2}+\mathrm{b}_{1} \beta_{\mathrm{r}}+\left(\mathrm{c}_{1}-|\mathrm{AP}|^{2}\right)=0
$$

After the required precision in the value of $\beta_{\mathrm{r}}$ is attained, the other dependent angles can be calculated which in turn provide the lead screw displacements $d 1$ and $d 2$ allowing a solution to the inverse kinematics. In most cases of fingertip position, the implementation is found to converge to the desired accuracy (less than a $\mathrm{mm}$ ) in just three iterations.

By Fichter's theorem [16] the finger should have at least twelve solutions to the inverse kinematics; however, many of these are not real due imaginary values of inverse trigonometric functions within the kinematic equations. Of the four possible real solutions, only one provides the valid configuration to the finger linkages as shown in Fig. 9. Allowing only a limited displacement of the lead screws, as shown in first case, ensures that a unique linkage configuration is obtained for a given fingertip position. The displacement range for such 
configuration is found to be $6 \mathrm{~mm}$ to $30 \mathrm{~mm}$, over which the finger has a unique solution to inverse kinematics. This is used to ensure non-singular configurations of the finger mechanism within its operational workspace for implementation of control algorithms.

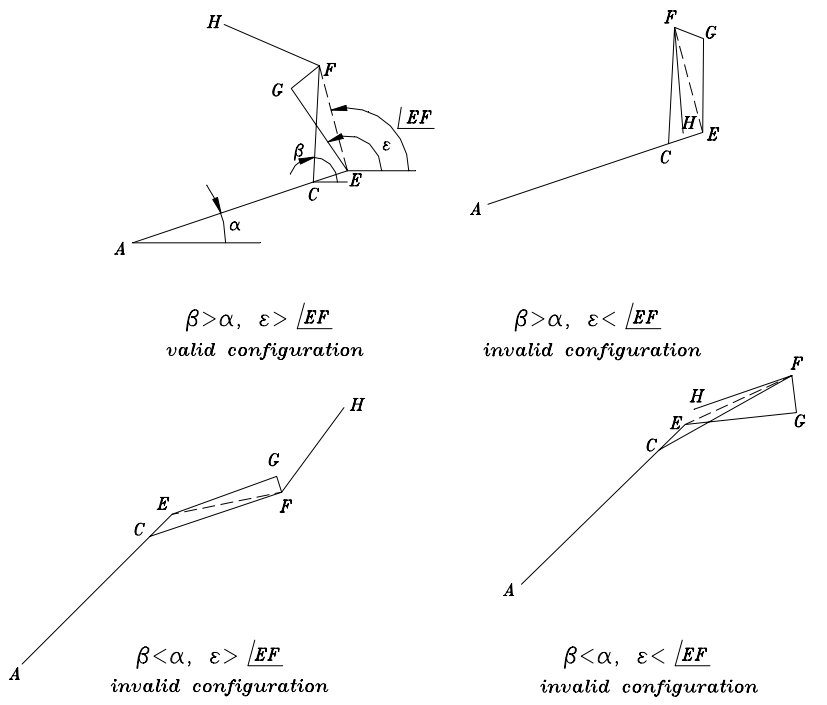

Fig. 9. Linkages configuration analysis

\section{FORCE ANALYSIS}

In order to design components of the finger, force analysis of the finger has been carried out. Equilibrium equations are derived from the free body diagram of each link. The four links, the three main finger sections and the bell crank, provide a total of twelve equations in twelve unknown forces, thus the equations are soluble. In actual design, however, each finger section has been loaded by variable forces at different inclinations to identify the critical condition of finger loading, also friction within the system has been accounted at various stages of the design [14].

The analysis of the finger gives,

$$
[\mathbf{A}][\mathbf{B}]=[\mathbf{C}]
$$

Where $[\mathbf{A}]$ is the matrix of geometrical coefficients, $[\mathbf{B}]$ is the column vector of the unknown forces, and $[\mathbf{C}]$ is the column vector of known forces with geometrical coefficients.

Inverting the above relation gives the unknown forces. These forces have been used to determine the loading conditions of various finger components. Fig. 10 shows the computed normalized force on the joint pins when a normal force is applied to the fingertip. The plots are shown in lead screws displacement range of $0.006 \mathrm{~m}$ to $0.03 \mathrm{~m}$. This displacement ensures uniform force variations as well as continuous fingertip motions without any singularity for the structure of the finger linkages. Beyond this range, the finger configuration is unattainable due to the dimensional constraints of the linkages, and the force variation becomes non-uniform with very high forces on the finger components. Within this operating range, the maximum force on any component is found to be within twenty times the fingertip force. The critical components identified are joint $\mathrm{A}$ and link $\mathrm{A}$ where the maximum resultant forces acting are twenty and sixteen times of the applied fingertip force respectively. The forces on these components are, however, maximum at $d 1_{\text {min }}, d 2_{\text {min }}$, which is the fully extended finger situation, making approximately $90^{\circ}$ angle with the vertical (lead screw) axis. This is clearly not the situation when a finger would normally be loaded hence in practical cases the applied load would be smaller than this.
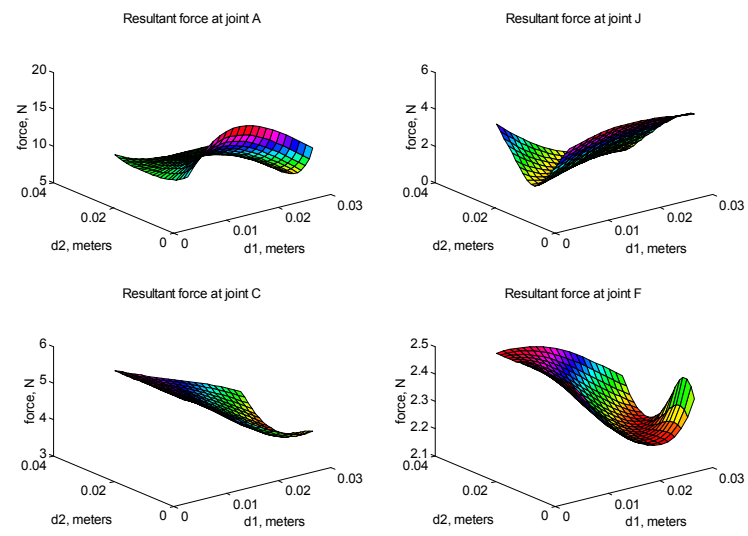

Fig. 10. Force in finger links for a precision grip

\section{CONCLUSIONS AND DISCUSSION}

This paper has presented design of an articulated finger mechanism suitable for adaptive end effectors. The mechanism removes a number of significant problems experienced with tendon-based designs. The finger actuation mechanism forms a compact and positive drive unit within the end effector's body with the use of solid mechanical linkage and the transmission through the toothed belts, thus offering a strong and reliable system for use in nuclear/hazardous environments where safety is an important consideration.

The control of the finger is easier with one motor and three brakes as compared to individually actuated finger joints, since only one motor needs to be controlled with brakes operating in just ON/OFF situation. Further the fingers can be driven in adaptive as well as precise control mode which can be rotated about its own axis allowing it to form either concentric or two fingers and opposing thumb grasps. Thus the finger design offers a practical solution to the specific tasks of grasping objects of specified shape and size securely within the structure of the end effector.

The range of the fingertip loci derived from the kinematic analysis provides the size of the object that can be grasped by the end effector using the finger. The continuity of the finger workspace proves the mechanical integrity of the system, which means any point in this region can be attained by the finger. The developed inverse kinematic solution for the complex geometry of the articulated finger allows the finger to be moved to a known location to grasp or to form a grasp-posture for the known shape and size of the object. The solution shows that for specified displacement of the lead screws, the finger has a unique solution to the inverse kinematics, thus there is no mathematical ambiguity in locating the fingertip. The numeric solution to the inverse kinematics converges fast which means real-time operation of the end effector is possible. 
The static analysis identifies the critical loading conditions of finger components based on which they can be designed. Further the analysis helps in sizing various end effector components like motors and electromagnetic brakes used in the design.

\section{ACKNOWLEDGMENTS}

The Faculty of Engineering \& Applied Science, Department of Electrical Engineering of University of Southampton, and the Overseas Research Student award from the UK Committee of Vice-Chancellors and Principals have supported this research. The authors acknowledge the contribution of the Central Design Service, in particular Dave Whatley for the realization of the design, and the construction of the prototype actuator mechanism.

\section{REFERENCES}

[1] Okamura, A. M., Smaby, N. and Cutkosky, M. R., 2000, "An overview of dexterous manipulation" Proceedings 2000 IEEE International conference on Robotics and Automation, San Francisco, pp. 255-262.

[2] Crowder, R. M., 1991, "An anthropomorphic robotic end effector" Robotics and Autonomous Systems, Vol. 7, pp. 253268.

[3] Crowder, R. M., Dubey, V. N., Chappell, P. H. and Whatley, D. R., 1999, "A multi-fingered end effector for unstructured environments" Proceedings of the IEEE Intl. Conf. on Robotics and Automation, Detroit, Michigan, Vol. 4, pp. 3038-3043.

[4] Cutkosky, M R., 1989, “On grasp choice grasp models and the design of hands for manufacturing tasks", IEEE Transactions on Robotics and Automation, Vol. 5. No 3, pp. 269-279

[5] Omata, T. and Nagata, K., 2000, "Rigid body analysis of the indeterminate grasp force in power grasps", IEEE Transactions on Robotics and Automation, Vol. 16, No. 1, pp. 46-54.

[6] Salisbury, J. K. 1985, "Design and control of an articulated hand", In M. T. Mason and J. K. Salisbury edited, Robot hands and mechanics of manipulation, MIT Press, Cambridge, MA, pp. 151-167.

[7] Jacobsen, S. C., Wood, J. E., Knutti, D. F. and Biggers, K. B., 1986, "The Utah/MIT dextrous hand: work in progress", In D.T. Pham and W.B. Heginbotham edited Robot Grippers, pp. 341-389.

[8] Bekey, G. A., Tomovic, R. and Zeljkovic, I. 1990, "Control architecture for the Belgrade/UCS hand", In S.T. Venketaraman edited Dextrous Robot Hands.: Spriger-Verlag New York pp. 136-149.

[9] Okada, T., 1986, "Computer control of multijointed finger system for precise object handling", In D.T. Pham and W.B. Heginbotham edited Robot Grippers, pp. 391-417.

[10] Ulrich, N., Paul, R. and Bajcsy R., 1988, “A mediumcomplexity compliant end effector" Proc. IEEE Intl. Conf. on Robotics and Automation, pp. 434-436.

[11] Doll, T. J. and Schneebeli, H. J. 1988, "The Karlsruhe hand" Proc. on IFAC robot control, Karlsruhe, pp. 383-388.

[12] Lin, L. R. and Huang, H. P., 1996, "Integrating fuzzy control of the dexterous National Taiwan University (NTU) hand", IEEE/ASME Transactions on Mechatronics, Vol. 1, No. 3, pp. 216-229.

[13] Jongkind, W., 1993, "Dextrous gripping in a hazardous environment guidelines, fault tolerance and control" Proc. IEEE Intl. Conf. on Systems Man and Cybernetics, Vol. 1, pp. 509-514.

[14] Dubey, V. N., 1997, "Sensing and control within a robotic end effector", PhD Thesis, University of Southampton, UK.

[15] Asada, H. and Slotine, J. J. E., 1986, Robot analysis and control, John Wiley, New York.

[16] Hunt, K.H., 1978, Kinematic Geometry of Mechanisms, Clarendon Press, Oxford. 